\title{
Randomized Prospective Trial of Arthroscopic Rotator Cuff With or Without Acromioplasty: No Difference in Patient-reported Outcomes at Long-term Follow-up
}

Brian Robert Waterman, MD¹, Jonathan Newgren, MA², Anirudh K. Gowd, BS², Brandon C. Cabarcas, BS², Bernard R. Bach, MD², Brian J. Cole, MD, MBA ${ }^{2}$, Anthony A. Romeo, MD², Nikhil N. Verma, MD ${ }^{2}$

${ }^{1}$ Wake Forest University School of Medicine, Winston Salem, NC, USA, ${ }^{2}$ Midwest Orthopaedics at Rush, Chicago, IL, USA.

Objectives: To evaluate long-term clinical outcomes after arthroscopic rotator cuff repair with and without acromioplasty.

Methods: Between 2007-2011, prospectively-enrolled patients undergoing arthroscopic repair for full-thickness rotator cuff tears were previously randomized into either acromioplasty or non-acromioplasty groups. Patients with death, advanced neurologic conditions, or subsequent shoulder arthroplasty were excluded. Baseline and long-term follow-up questionnaires, including the American Shoulder and Elbow Surgeons (ASES), Simple Shoulder Test (SST), University of California-Los Angeles (UCLA), Visual Analog Scale (VAS) for pain, and Constant scores were obtained. Rates of revision rotator cuff surgery, or secondary reoperation were recorded. Averages with standard deviation (SD) were calculated, and t-tests were utilized to compare outcomes of interest between cohorts.

Results: After exclusion of 5 additional patients from the short-term follow-up study, 66 of 90 patients (73.3\%) were available at 92.4 months $( \pm 10.5)$. Comparison of baseline demographics and intraoperative information revealed no significant differences, including age, gender, workers compensation, acute mechanism of injury, tear size, degree of retraction, and surgical technique (e.g. single- vs. double-row). At final follow-up, there were no statistically significant differences according to ASES $(p=0.33)$, VAS pain $(p=0.79)$, Constant $(p=0.17)$, SST $(p=0.05)$, UCLA $(p=0.19)$, and SF-12 $(p=0.79)$ in patients with and without acromioplasty (Figure 1). One patient with acromioplasty $(2.9 \%)$ and two patients without acromioplasty $(6.3 \%)$ sustained atraumatic recurrent rotator cuff tear with secondary repair $(p=0.99)$.

Conclusion: Combined acromioplasty and rotator cuff repair offer no significant long-term benefits in patientreported outcomes or secondary surgery when compared to arthroscopic rotator cuff repair alone. 


\section{Patient-Reported Outcomes in Rotator Cuff Repair with or without Acromioplasty}

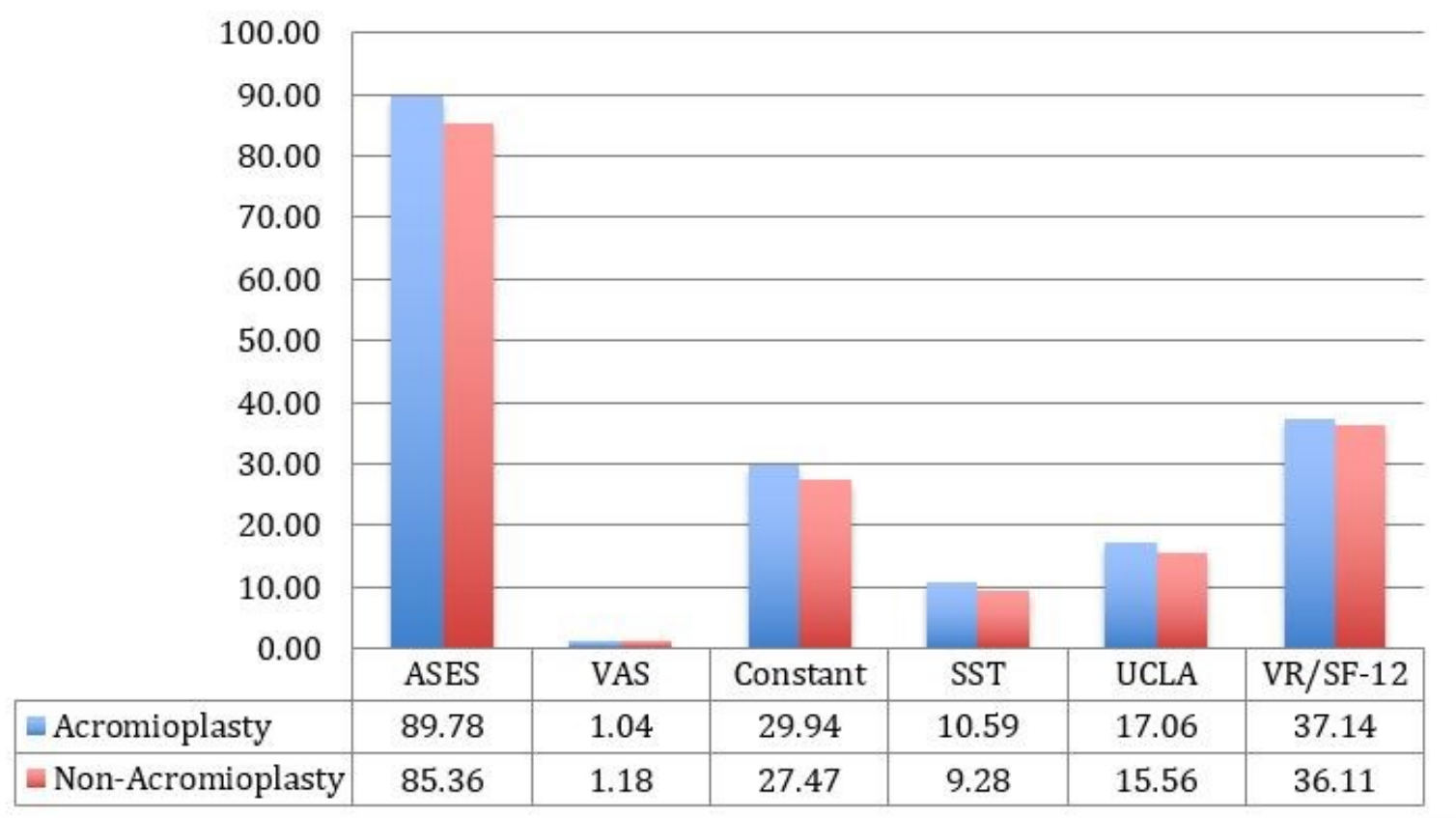

Figure 1

The Orthopaedic Journal of Sports Medicine, 6(7)(suppl 4) DOI: $10.1177 / 2325967118 S 00080$

(C)The Author(s) 2018 